\title{
Improvement of Zero Suspension Force Performance Using a Special Magnet for a Magnetic Suspension Device
}

\author{
Feng Sun ${ }^{1, a}$, Koichi Oka ${ }^{2, b}$ and Junjie Jin $^{3, c}$ \\ ${ }^{1}$ No.111, Shenliao West Road, Economic \& Technological Development Zone, \\ Shenyang, 110870, P.R.CHINA \\ ${ }^{2} 185$ Miyanokuchi, Tosayamada, Kami city, Kochi 782--8502, Japan \\ asunfeng2009@gmail.com (corresponding author), boka.koichi@kochi-tech.ac.jp
}

Keywords: Zero suspension force, Magnetic suspension, Permanent magnet

\begin{abstract}
This paper proposes an improvement method of zero suspension force performance using a special permanent magnet for a magnetic suspension device. The zero suspension force of a permanent magnetic suspension device is indispensable for floating off when the suspended object is stuck on accidentally. Generally, the magnetic suspension system using permanent magnet is difficult to realize the zero suspension force, since the permanent magnet cannot make its attractive force zero. However, the proposed magnetic suspension device has realized the semi-zero power suspension and semi-zero suspension force, which mainly consists of a disk-shape permanent magnet, two " $F$ " shape iron cores, a suspended object, and an actuator. In the magnetic suspension device, since there is magnetic leakage, the suspension force could not be made exact zero. Therefore, in this paper, the zero suspension force will be improved using a special shape permanent magnet. The comparison results indicate that the zero suspension force is decreased about 80 percent.
\end{abstract}

\section{Introduction}

Until now, many magnetic suspension devices have been proposed, and applied in many fields [1]. Most of these magnetic suspension devices are using electromagnet whose attractive force can be controlled by its current. We call the attractive force used to suspend an object or a device as the suspension force in the magnetic suspension system. When the current is zero, the suspension force of the electromagnetic suspension (EMS) device is zero. The zero suspension force of magnetic suspension device is indispensable for floating off when the suspended object is stuck on accidentally. However, the EMS has some disadvantages of heat generation, high-energy consumption, big coil size, and so on.

With the development of magnetic material, the attractive force of the permanent magnet becomes very strong [2]. Many researchers focus on developing magnetic suspension systems using permanent magnets. Morishita and Azukizawa have proposed a zero-power magnetic suspension system using the hybrid electromagnet with the permanent magnet and the coil [3], but the system had to use the large countercurrent to counteract the attractive force of the permanent magnet, when the system needed the zero suspension force. For permanent magnetic suspension system, Mizuno et al. have proposed a zero-power magnetic suspension system with a permanent magnet and three flux-path control modules [4]. Ueno and Higuchi presented a zero-power magnetic levitation technique using a composite of magnetostrictive and piezoelectric materials [5]. The authors have proposed a hanging type magnetic suspension system using a permanent magnet and an actuator [6]. All the permanent magnetic suspension systems mentioned above are difficult to generate the zero suspension force.

However, the authors also have proposed a magnetic suspension system using a disk-shape permanent magnet and a rotary actuator [7]. In this system, the suspension force was controlled by a variable flux path control mechanism, and the zero suspension force could generate in some conditions, theoretically. Whereas there was magnetic leakage, the zero suspension force was not zero exactly. 
Therefore, this paper proposes an improvement method to decrease the zero suspension force of the original magnetic suspension system. In this paper, the magnetic suspension device and the suspension principle are introduced, and the improvement method is proposed. According to the flux field analysis results and the comparison experimental results, the performance of the improvement device is examined.

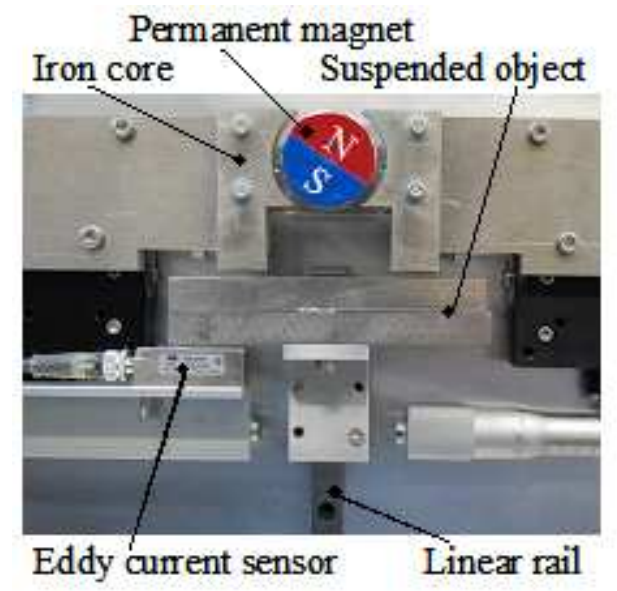

Fig. 1. Experimental prototype

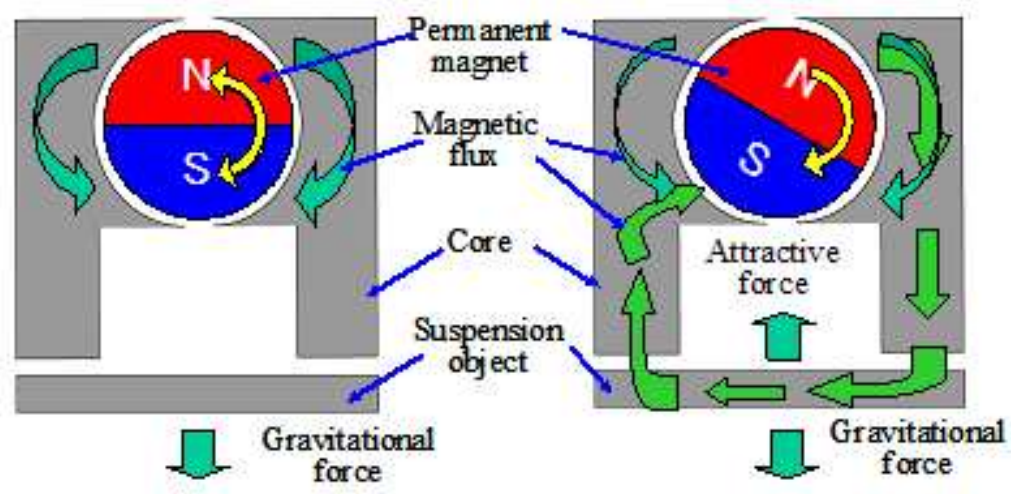

(b)

Fig. 2. Suspension principle

\section{Experimental Prototype and Suspension Principle}

Experimental Prototype. The proposed experimental prototype is shown as Fig.1. This prototype consists mainly of a disk-type permanent magnet, a rotary actuator containing a gear reducer and an encoder, a pair of opposite "F" shape permalloy cores, a suspended object and an eddy current sensor. The magnet that is located in the opposite " $\mathrm{F}$ " shape cores is a neodymium magnet and magnetized in radial direction. The diameter of the magnet is $30 \mathrm{~mm}$ and the thickness is $10 \mathrm{~mm}$. A rotary actuator behind of the magnet drives the magnet rotate. The actuator that has an encoder measuring the angle of the magnet cannot be seen in Fig. 2. The thickness of the two cores is same as the magnet. The suspended object is installing on a linear rail, and can move in the vertical direction only.

Suspension Principle. The suspension principle can be understood from Fig. 2. This figure shows a schematic diagram of a disk permanent magnet, two opposite F-type iron cores and a suspended object. In order to understand easily, we assume that there is no flux leakage to the air in this magnetic suspension system. Fig. 2 (a) shows that the magnetic poles of the magnet are aligned in the vertical direction, and the $\mathrm{N}$ pole is at the upper side and the $\mathrm{S}$ pole is in the lower side. In this case, the facing angle of the $\mathrm{N}$ pole and $\mathrm{S}$ pole to each core are same, so all magnetic flux comes from the $\mathrm{N}$ pole and is absorbed into the $\mathrm{S}$ pole through each core respectively. There is no flux flowing through the suspension object, so zero attractive force generates between the cores and the levitated object. Therefore, the zero suspension force can be realized in this situation. However, Fig. 2 (b) shows the magnet rotated a certain angle, the facing angle of the $\mathrm{N}$ pole becomes bigger than the $\mathrm{S}$ pole in the right core, and that is reverse in the left core. Since that, the flux from the N pole in the right core is more than that in the left core. Some of the flux in the right core flow through the suspension object to the left core and is absorbed by the S pole. Consequently, there are some flux flowing through the levitated object, and the attractive force is generated. The flux flowing through the levitated object becomes more as the rotated angle is larger, until the rotated angle reaches 90 degrees.

\section{Existing Problem and Improvement Method}

Existing Problem. In order to understand the suspension force performance of the magnetic suspension device, the attractive force between the suspended object and two iron cores was measured with a load sell, when the air gap length varied from $2 \mathrm{~mm}$ to $8 \mathrm{~mm}$. The measured results of attractive force are shown in Fig.3. Fig.3 shows the relationship between the attractive force and the rotational angle of magnet. In the figure, the force is expressed when the rotational angle of 


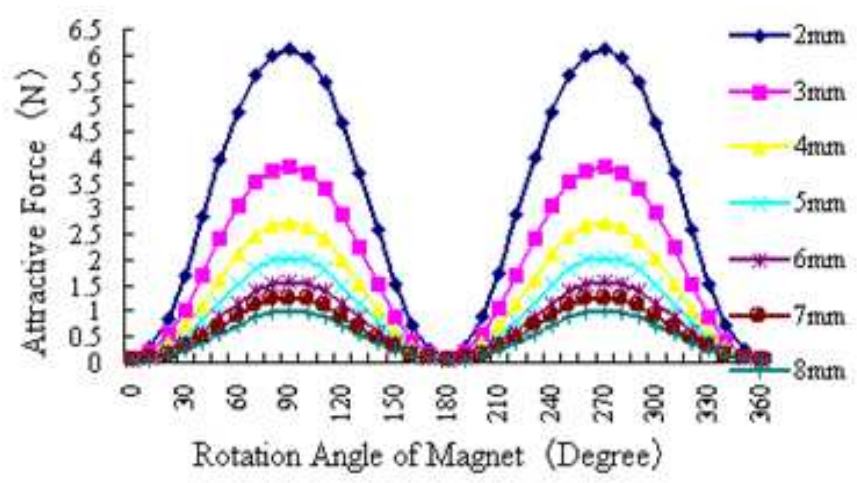

Fig. 3. Attractive force between cores and suspended object using original magnet

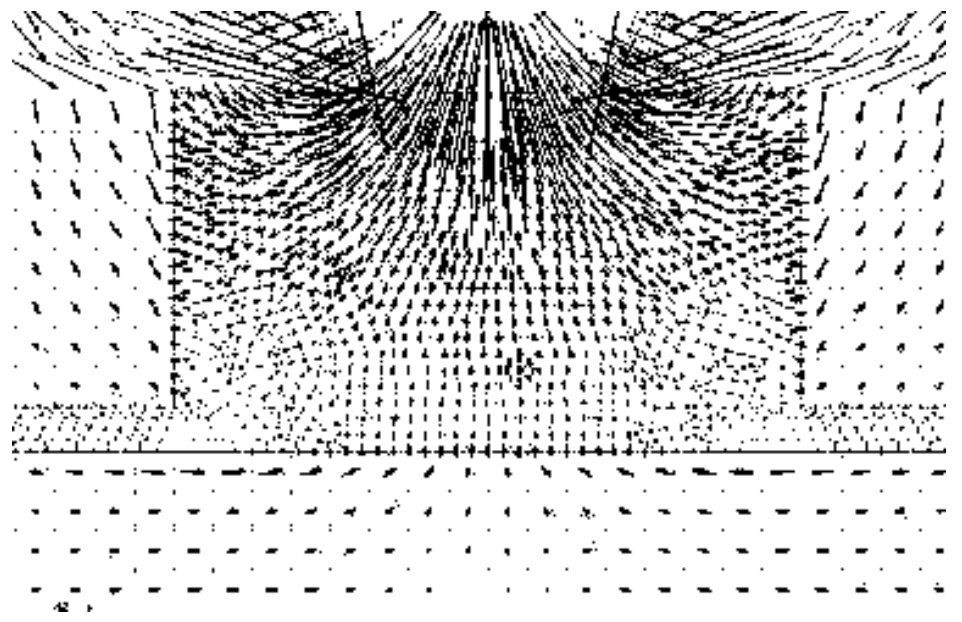

Fig. 4. Magnetic flux field analysis of original device using IEM

magnet is changing and the length of air gap is seemed as a parameter. The results indicate that, the attractive force varies as the rotational angle of magnet changes, and smaller distances yield greater attractive force. Moreover, when the rotational angle is around 0 and 180 degrees, i.e. the $\mathrm{N}$ pole and $\mathrm{S}$ pole stop at directly above or below, the attractive forces are approaching to zero, but not zero.

In order to understand the reason that the suspension force is not zero, the magnetic flux field of the device was analyzed using IEM (Integral Element Method) analysis. The analysis result is shown in Fig.4. In the figure, the direction of arrows express the direction of the magnetic flux, and the size of the arrows express the intensity of the magnetic flux density. The magnetic flux that comes from $\mathrm{N}$ pole of the magnet almost returns to the $\mathrm{S}$ pole only through the upper side of the iron cores, and there is a little part of magnetic flux flows through the lower side of the iron cores, the suspended object, and the air space, finally returns to the S pole. Therefore, the attractive force between the iron cores and the suspended object is not zero. This problem is caused by the magnetic leakage between the magnet and the suspended object.

Improvement Method. According to the analysis above, many improvement methods can be used to decrease the magnetic leakage between the magnet and the suspended object. In this paper, we just discuss the improvement method using a special shape permanent magnet shown in Fig.5. Fig.5 shows the size of the special magnet, and its thickness is $10 \mathrm{~mm}$.

Fig.6 shows the analysis results of magnetic flux field of the special magnet. The results show that the magnetic flux density varies as falling off between two pick periods, which is similar to the shape of the saddle. Therefore, if using this special magnet to replace the original round magnet, when the magnetic poles of this special magnet stop at the vertical direction, the leakage of the magnetic flux from the magnetic poles will be much less than that in original mechanism using the round magnet. 


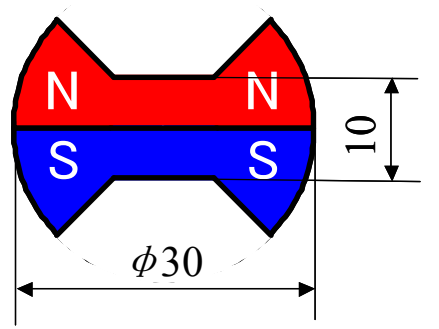

Fig. 5. Special shape permanent magnet

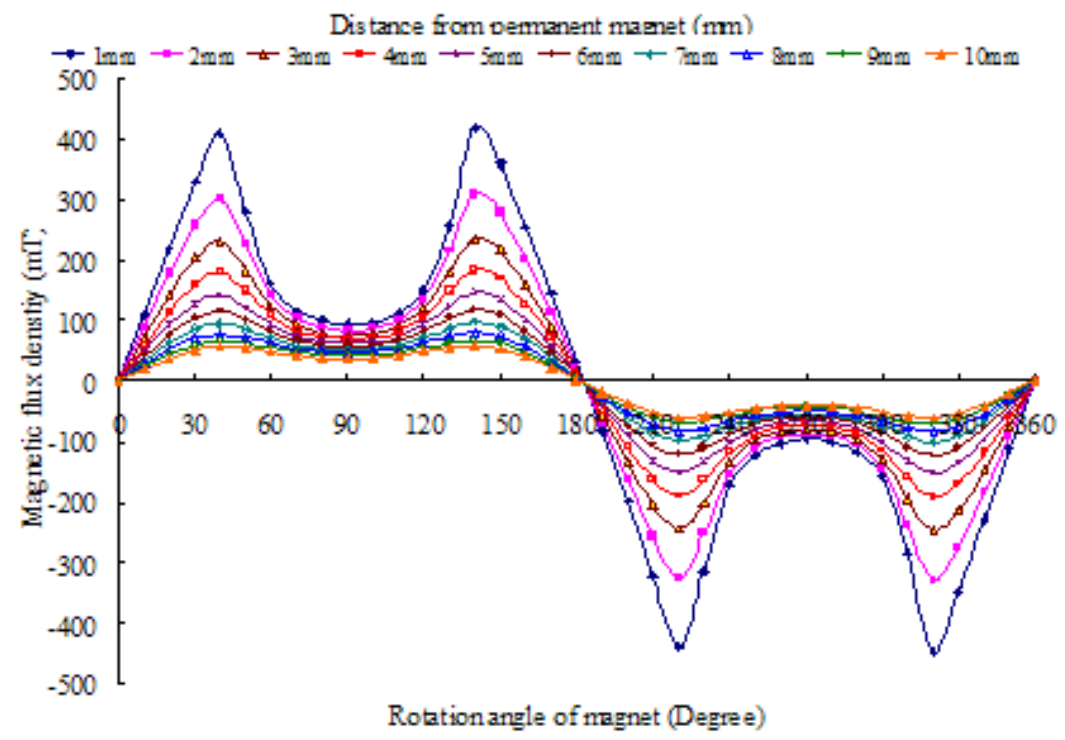

Fig. 6. Magnetic flux density of the special magnet

\section{Performance of Improved Device}

Suspension Force. In order to know the suspension force performance of the improved device using the special permanent magnet that has the magnetic performance shown as Fig.6, the attractive force between the iron cores and the suspended object was examined by the measurement experiment. The examined results are shown in Fig.7. The attractive force from the iron cores also resembles to the results of the original device, but the graph shape becomes thin, and the linearity of the attractive force becomes good towards to the rotational angle of the magnet. The results indicate that the rotation of the angle of the special magnet can control the suspension force, and the special magnet does not change the force performance of the magnetic suspension device. Moreover, it can be seen that the attractive forces at 0 degree and 180 degrees are smaller than the results of the original device.

Magnetic Flux Field. Additionally, the magnetic flux field of the improved device was analyzed using analysis. The analysis result is shown in Fig.8. The size of the arrows corresponding to the magnetic flux density in this figure is same with that in Fig.4. However, the arrows almost cannot be seen in Fig.8, which indicate that the suspension force between iron cores and the suspended object is almost zero.

Comparison Results. In order to evaluate the improvement method, the zero suspension forces between the original device and the improved device were examined, when the magnet stopped at 0 degree. The comparison results are shown in Fig.9. From the results, we can see that, the zero suspension forces of the improved device are much smaller than the results of original device, and the zero suspension force decreases about 80 percent of that of the original device. Therefore, the improvement method using a special shape permanent magnet is available, and the improvement effect is conspicuous. 


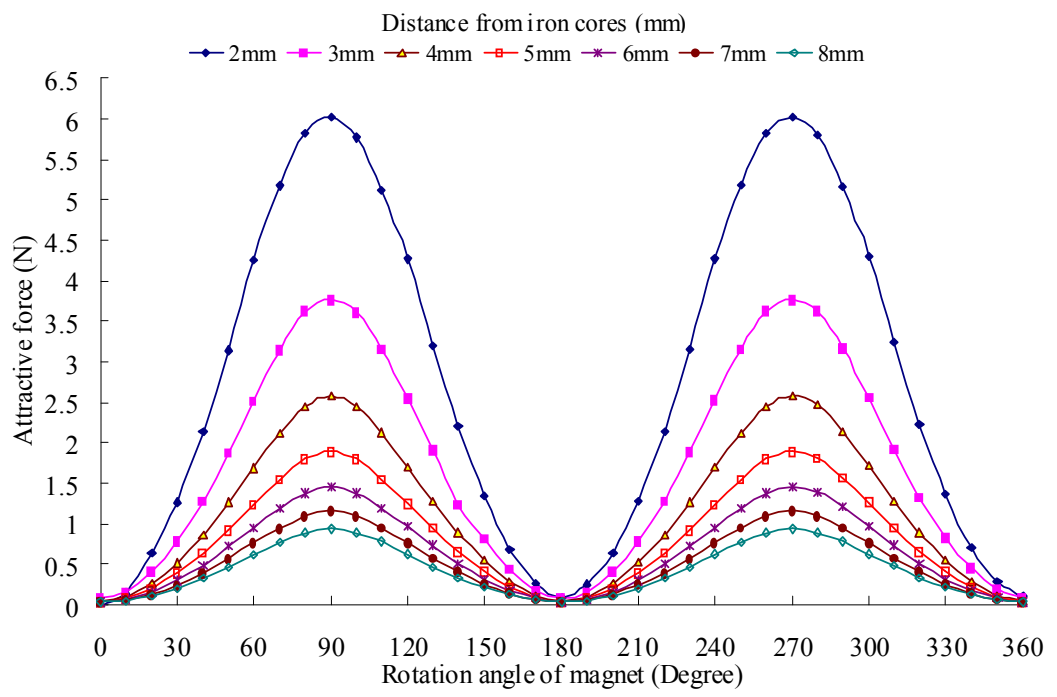

Fig.7. Attractive force between cores and suspended object using special magnet

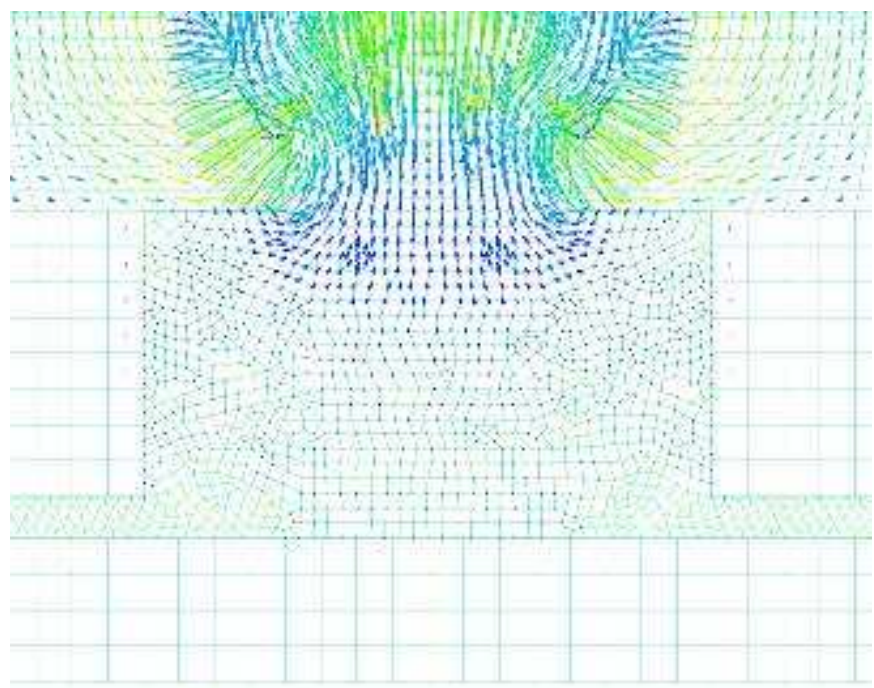

Fig. 8. Magnetic flux field analysis of improved device using IEM

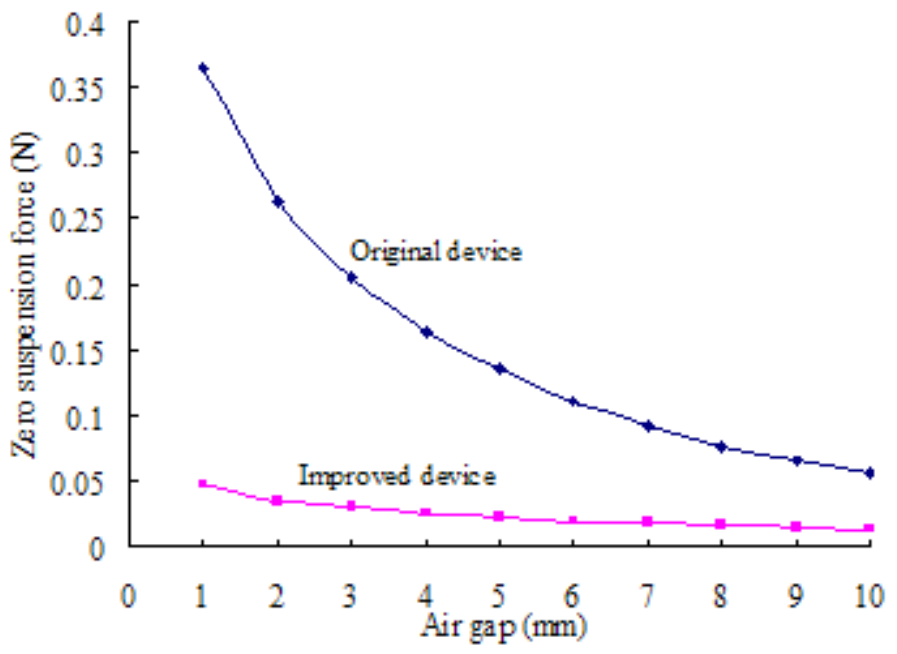

Fig. 9. Comparison results of zero suspension force 


\section{Summary}

This paper introduced a magnetic suspension device and the suspension principle, analyzed the reason that the zero suspension force is not zero, and obtained the reason was the magnetic leakage from the magnet to the suspended object. An improvement method using a special shape permanent magnet was proposed, the performance of the improved device was examined. The results indicated that the zero suspension force of the improved device decreased about 80 percent of that of the original device. Therefore, the better zero suspension force performance could make the suspended object floating off easily, when the suspended object was stuck on accidentally.

\section{References}

[1] G. Schweitzer and E.H. Maslen, Magnetic Bearings: Theory, Design, and Application to Rotating Machinery, Springer Dordrecht Heidelberg London New York, 2009.

[2] D.N. Brown, B. Smith, B.M. Ma and P. Campbell. The dependence of magnetic properties and hot workability of rare earth-iron-boride magnets upon composition, IEEE Transactions on Magnetics, 40 (2004) 2895-2897.

[3] M. Morishita and T. Azukizawa, Zero power control method for electromagnetic levitation system, Trans. IEE of Japan, 108 (1988) 447-454.

[4] T. Mizuno, Y. Hirai and Y. Ishino, Flux-path control magnetic suspension system using voice coil motors, Journal of System Design and Dynamics, 1 (2007) 147-158.

[5] T. Ueno and T. Higuchi, Zero-power magnetic levitation using composite of magnetostrictive/piezoelectric material, IEEE Transactions on Magnetics, 437(2007) 3477-3482.

[6] F. Sun and K. Oka, Zero power control for hanging type maglev system with permanent magnet and VCM, Trans. of JSME Series C, 75 (2009) 1383-1388.

[7] F. Sun and K. Oka, Development of noncontact suspension mechanism using flux path control by disk magnet rotation, Trans. of JSME Series C, 76(2010) 2976-2981. 